\title{
The Intelligent Control Technology of Manufacturing Execution Process Based on RFID
}

\author{
Ruicheng Fan ${ }^{1, a}$, Aimin Wang ${ }^{1}$ and Yanpeng Lv ${ }^{1}$ \\ ${ }^{1}$ Beijing Institute of Technology, Beijing of China
}

\begin{abstract}
Intelligent manufacturing is an important trend in the development of the future manufacturing industry, and how to use the current technology to realize the existing traditional manufacturing mode change to the intelligent manufacturing mode need to be studied. Aimed at the implementation of the intelligent manufacturing mode, this paper proposed the intelligent manufacturing execution process control technology based on RFID. The framework of intelligent manufacturing execution process control technology proposed an effective way to realize the manufacturing process intelligent control based on existing MES; the technology of logistics identification and control based on RFID realized the effectiveness control of logistics; the intelligent monitoring technology of mobile terminal solve the mobile terminal positioning problem, MES pushing information to mobile terminal problem and other problems. Finally, the author developed a set of intelligent manufacturing execution process control system which includes RFID reader, MES server, mobile terminal, and verified the effectiveness of the above mentioned technologies
\end{abstract}

\section{Introduction}

In recent years, with the development of intelligent perception and industrial Internet technology, intelligent manufacturing has become a new trend in the traditional manufacturing industry. The separating of information and physical entities is one of the most serious problems in the traditional manufacturing execution system, but the intelligent sensing technology which is based on radio frequency identification technology can solve the problem effectively, it provides an effective means to make the manufacturing more automatic and intelligent. In order to realize the intelligent control of manufacturing execution processes and realize the efficient control of merging the production process information data and physical products, it is necessary to upgrade the means of traditional manufacturing processes control.

Since the concept of "intelligent manufacturing" is proposed, more and more domestic and foreign scholars have studied the various fields of intelligent manufacturing.

In the application of RFID, Reza Vatankhah Barenji put forward a frame which can be used to deploy the RFID distributed monitor and control system. It is responsible for different control and monitoring tasks by a group of agents. At the same time, the agents can cooperate with each other to improve the manufacturing system's agility, flexibility and reconfigurability. In domestic, Yangyi of Huazhong University of Science and Technology designed a digital warehouse manage system that is based on RFID.
Manufacturing Execution System is an essential part to realize the informatization control of workshop product processes. Zhang Caifang the analyzed on the characteristics of workshop logistics of the discrete manufacturing industry, and proposed the architecture of the logistics lean management system for MES.

For the application of RFID in MES system, many scholars have done a lot of research. Wang Jing et al. researched on the optimization model of the logistics information platform based on RFID for manufacturing execution system, and emphasized its importance to the development of enterprise. On the basis of analyzing the application requirements and application technology of RFID, Zhang Yangyang designed a real time data acquisition system of workshop.

It can be seen from the above research background, RFID and manufacturing execution systems have been extensively studied, however, how to use RFID technology to realize the intelligent control of manufacturing execution process still needs to be further studied.

Firstly, this paper analyzes the key technology of realizing the intelligent control of manufacturing process. Secondly, the framework of intelligent control technology of manufacturing processes is proposed. Thirdly, this paper studied the technology of logistics recognition which is based on RFID and the technology of mobile terminal intelligent information pushing.

\section{Technology challenge}

\footnotetext{
$\overline{{ }^{\mathrm{a}} \text { Corresponding author: fanruicheng } @ 163 . c o m}$
} 
After analysis, the following three aspects of technology need to be studied to make manufacturing process control intelligent and monitoring of the execution flexible.

1. Research on the framework of intelligent manufacturing execution process control technology

In this research point, the framework of intelligent manufacturing execution process control should be proposed by analyzing the intelligent sensing application mode in production process, and combining with the manufacturing execution system.

2. The technology of logistics identification and control based on RFID

Firstly, the technology takes information data of MES as a support. Secondly, the technology is based on RFID. Finally, by analyzing the execution data, the technology can realize the intelligent transfer control of logistics.

3. The technology of mobile terminal intelligent information pushing

By using RFID, web service and other technologies, this technology can push the device information to the mobile terminal and realize the feedback of execution information from mobile terminal.

\section{The framework of intelligent manufacturing execution process control technology}

Intelligent manufacturing execution process control technology mainly studies how to combine the RFID technology, mobile terminal technology with traditional MES, and it proposes a smart manufacturing mode.

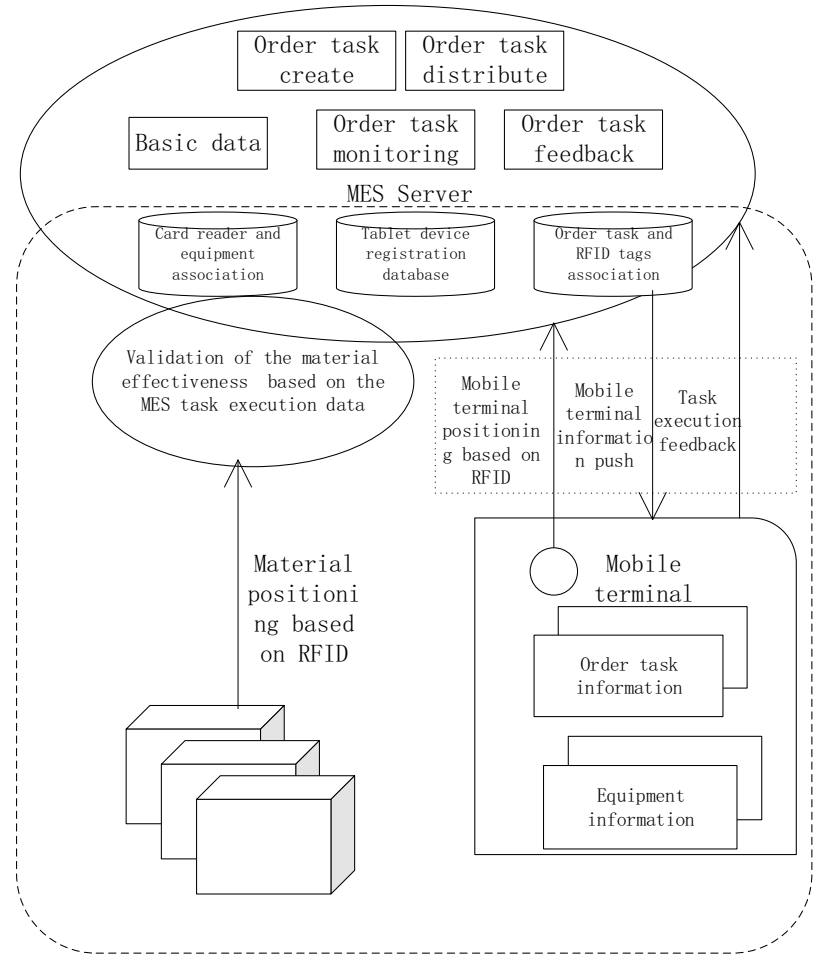

Figure 1. Technology Frame

As shown in Fig.1, the intelligent manufacturing execution process control technology mainly includes the intelligent logistics identification and its availability control technology and the mobile terminal intelligent information push technology. Its main applications are as follows:

1. The core of the manufacturing execution control technology is MES, which includes task order create、 task order technical preparation、 task order distribution and task order execution and so on . Then, the intelligent control technology carries out the modular expansion which is based on the existing MES system and achieves a certain degree of intelligent control finally.

2. The technology of logistics identification and control based on RFID is mainly used for material flow control in the stage of task execution. This technology is based on the RFID, and it takes the MES system task execution information as support. It achieves the material's intelligent flow control by the MES server's calculation.

3. Mobile terminal intelligent information push technology is based on XMPP protocol and web service technology, it uses the device-reader, tablet-RFID label these two relationships and combines with MES data to realize the instant MES information push.

\section{The technology of logistics identification and control based on RFID}

This technology mainly realizes the position of production material/workpiece, and its positioning datum is the machine tool equipment. That is to say, the technology can achieves the material reasonable position control on the present moment, the principle is as follows:

\subsection{MES related data preparation}

In order to achieve the positioning of task order by the RFID sensing technology, we need to prepare the relevant data in the MES system. Data preparation includes the following two aspects: machine tool equipment and RFID reader data association and task orders and RFID tag data association. After the establishment of these two associations, MES system uses RFID sensing technology to establish the relationship of RFID tags and RFID readers, thereby indirectly establishes the relationship of machine tool equipment and task order.

\subsection{Task position validity judgment}

The validity checking of the task order position is to determine whether the machine that the order arrives is reasonable and effective. The validity checking of the task position mainly consists of two steps: step1: to determine whether the label comes to the reader and meets the corresponding conditions of stay; step2, to determine whether the current order execution status is corresponding to the device information. Specific rules for a single label are as follows: 
1. Timer initialization

(1-1) set the timer trigger interval

Set Timer1, Timer2;

Timer1.interval $=6000$;

Timer2.interval $=300$;

(1-2) defining the timer processing function

Timer1's timing processing function is Timer1_Tick.

Timer2's timing processing function is Timer2 Tick

2. Timer trigger

Timer2 triggers the execution of Timer2_Tick, and the function will call reader control function 'Scan ()' for card readers scanning and reading. Timer1 triggers the execution of Timer1_Tick and the function mainly reset number of scan and push the information of tag's leave.

3. Location estimation rules

Take a card reader Reader1 as an example, this technology uses a dictionary 'reader1Dic' To store the number of tags that it reads and the number of times the tag is scanned in a time period.

(3-1) scan and count for a tag information

$\mathrm{Scan}() \rightarrow$ Getlabel1 $\rightarrow$ reader1Dic.label1.count +1

(3-2) determine whether the label is satisfied with the conditions of arrival

If (reader1Dic.label1.count $>15$ )

\{ function

LabelCome(label1);// Label arrival processing

$$
\text { \} }
$$

(3-3) the number of the tags scanned times clear and tags leave judgment

For (int $\mathrm{i}=0 ; \mathrm{i}<$ reader1Dic.count $; \mathrm{i}++)$

\{

If (reader1Dic[i].count $<10)$

\{

LabelLeave(readerDic[i].Value);

\}

reader1Dic $[\mathrm{i}]$.count $=0$;

\}

4. Association information acquisition

(4-1) get tag types from a tag database

GetLabelType(label1);

(4-2) access to the device information associated with the reader

GetEquipmentByReader(reader1) $\rightarrow$ equipment 1

(4-3)tags are about material

(4-3-1) access to the base information of the order by tags

GetOrderInfoByLabel(label1) $\rightarrow$ order 1

(4-3-2)get the information of order distribution and determine whether it arrived this device.

GetProcessByEquipment(order1,equipment1) $\rightarrow$ proce ss

(4-3-3) that the device has a distribution information corresponding to the order, then obtains the execution information of the previous procedure.

GetPrevProcess(process) $\rightarrow$ prevProcess

(4-3-4) determine the status of the pre order, if completed, then the material arrived the device availably, otherwise it is invalid.

If $($ prevProcess.completed $==$ true $)$

\{

UpdateProcessInfo(process);// Update the information of the current order on this device \}else \{\}

\section{Intelligent monitoring technology of mobile terminal}

Mobile terminal intelligent monitoring technology is based on RFID, and it combines with Android mobile devices push technology and web service technology. Finally, it realize the intelligent control of MES system. The realization principle of intelligent monitoring of mobile terminal is as follows:

\subsection{The control of mobile terminals' effectiveness}

In order to realize the intelligent monitoring by mobile terminal, a database of mobile terminal needs to be created in MES system. This database is used for mobile terminal management, including mobile terminal info create mobile terminal and RFID tag association and so on. At the same time, user authentication mechanism should be added when developing the app based on android platform, and the user data of mobile terminal and MES system should be unified. If you want to monitor the task in MES system, you need to use the mobile terminal registered in MES system and use the correct account to $\log$ in.

\subsection{Mobile terminal intelligent information push}

Mobile terminal intelligent information push mainly includes two points as follows:

A. mobile terminal positioning

Mobile terminal location positioning methods are same with the order task positioning in the sections above. Get the relationship of tags and readers by RFID technology, and then establish the position relationship between mobile terminal and machine tool.

B. Intelligent information push

When a mobile terminal reaches a machine tool, MES server determines whether it is effective. If it is effective, the MES server will push the information about this machine tool to the mobile terminal. The information about machine tool mainly includes the id of machine tool and the flag that indicates whether the terminal arrived or left. The mobile terminal will react when it receives the pushed information. According the flag, if the terminal is 'arrive', the terminal will request the information about machine tool and related task process information from MES server by machine tool id; if the terminal is 'leave', it needs to shut off the display interface automatically.

\subsection{Mobile terminal feedback the execution information of order task process}

In addition to receiving pushed information, the terminal also can view the task 、 feedback the task start or finish and other operation. The mobile terminal can select and view the process task information in the MES system or 
start finish the corresponding task through the MES server's web service.

\section{Application Verification}

On the basis of solving the above technologies, the author has developed a system for intelligent manufacturing execution control, which mainly includes the following aspects:

1. On the original MES system, the registration management module of the mobile terminal is added, and the relationship management module between the readers and machine tools is added.

2. The App based on android platform is developed.

3. The development and implementation of the intelligent push technology and the effective identification of MES server.

\subsection{MES related data management}

In order to realize the positioning of the material and the mobile terminal, the relationship between the two and the tags needs to be established firstly. In this system, two interface functions of the reader management and the tablet management are added to the original MES system,as shown in Fig.2 and Fig.3. They are used to create a card reader - device association and the tablet RFID label association. The association management of the material task and label is achieved by increasing the field in the order.

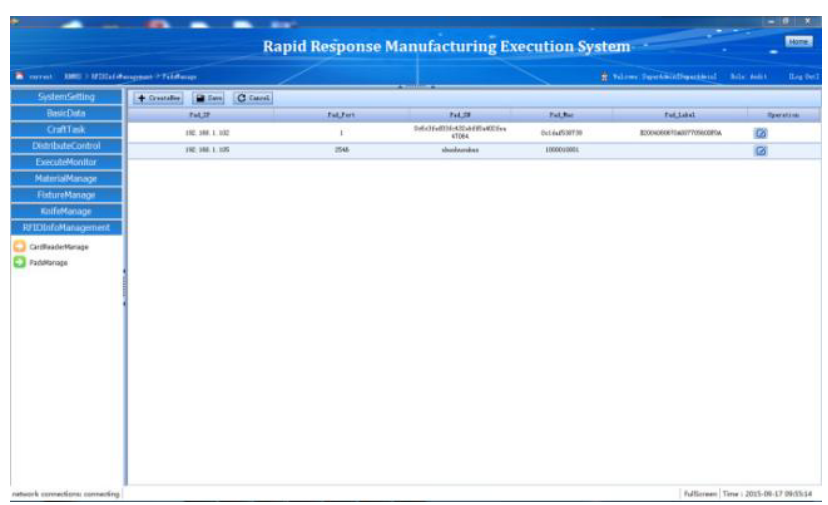

Figure 2. Mobile Terminal Management

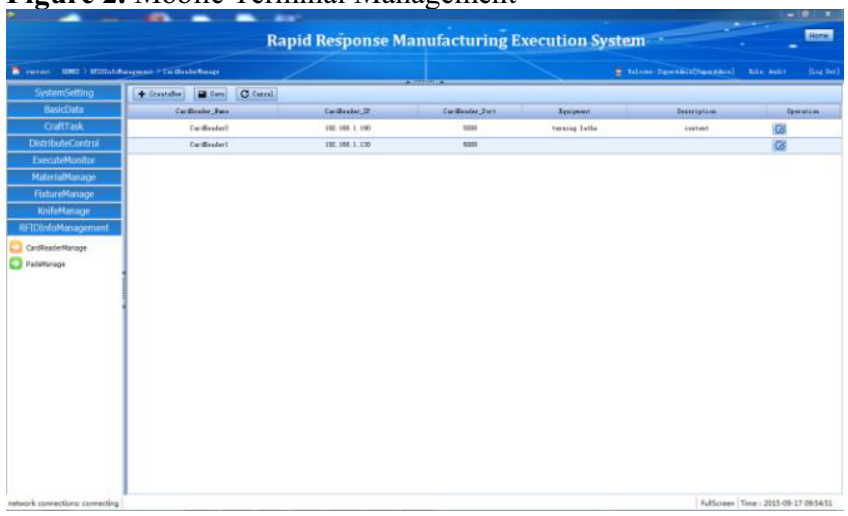

Figure 3. Card Readers Management

\subsection{APP development of mobile terminal}

In this system, we used the Xiaomi tablet from domestic manufacturers as a mobile terminal, and developed the app based on android 4.0 platform.

APP development of mobile terminal includes three aspects, namely, the development of the interface, operation data from web service, the development of information push based on the XMPP protocol.

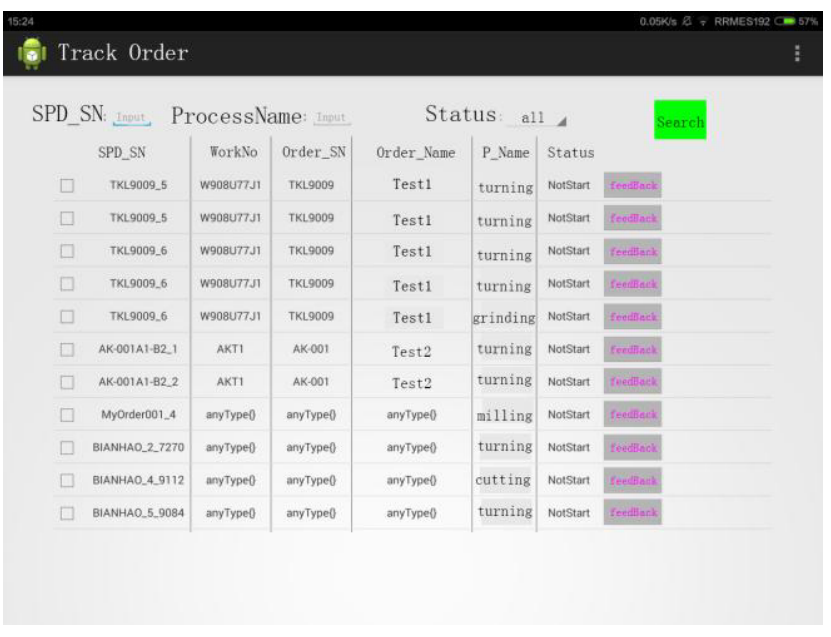

Figure 4. Query Tasks By WebService

\section{|ํ. FeedBack}

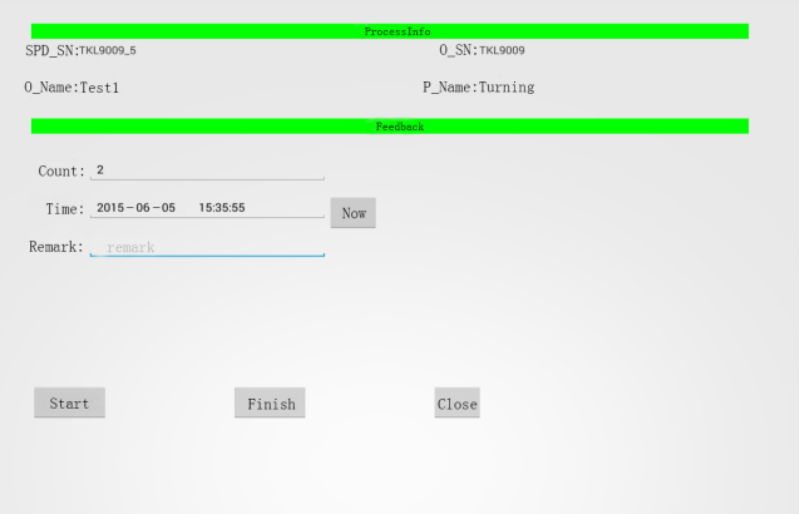

Figure 5. Feedback Execution Information

\subsection{MES server development}

This paper developed the intelligent push technology and the effective recognition technology based on the original MES system server. This paper achieved the reading of RFID tags based on the interface functions provided by the RFID manufacturers.Information push that based on XMPP protocol is launched by the MES server, through the Android PN server, and it arrived the tablet client finally.

\section{Conclusion}

In this paper, a framework of manufacturing execution process control based on RFID is proposed and it solved the logistics effectiveness control technology and 
intelligent push technology of mobile terminal. And then a set of intelligent manufacturing execution process control system including mobile tablet, RFID reader, MES server is established, it successfully achieved the logistic validity verification based on RFID, MES server push information to the mobile terminal, mobile terminal feedback information to MES server and other functions.

\section{Acknowledgment}

The research of this thesis is supported by the National Natural Science Foundation of China.

(Fund number: 51175045)

\section{References}

1. Reza Vatankhah Barenji, Ali Vatankhah Barenji and Majid Hashemipour. A multi-agent RFID-enabled distributed control system for a flexible manufacturing shop [J], The International Journal of Advanced Manufacturing Technology Vol.71 (2014).

2. Yang Yi, Digital warehouse management system based on RFID [D], Huazhong University of Science and Technology (2008).

3. Zhang Caifang and Yin Chao, Research on Logistics Lean Management System of Discrete Manufacturing Industry for MES [J], Modular Machine Tool \& Automatic Manufacturing Technique monthly No.8 (2014).

4. Wang Jing and Ma Rongquan, Implementation of the Logistics Information System Optimization Model of RFID-based Manufacturing [J], Journal of Modem Information Vol. 33 , No.8 (2013).

5. Zhang Yangyang and Chen Lin, Design and Implement of Discrete Manufacturing Plant RealTime Data Collecting System Based on RFID [J], Journal of Jiangnan University(Natural Science Edition) Vol.12 No.1 (2013). 